DOI: https://doi.org/10.14311/TPFM.2021.006

\title{
FINITE ELEMENT SIMULATION OF VISCOELASTIC FLUID-STRUCTURE INTERACTION
}

\author{
Alexander Drobny ${ }^{1}$, Elfriede Friedmann ${ }^{1}$ \\ ${ }^{1}$ Institute of Mathematics, University of Kassel, Heinrich-Plett-Straße 40, Germany
}

\begin{abstract}
The research of fluid-structure interaction (FSI) problems is a continuously growing field. We present a numerical solution method for viscoelastic FSI problems employing the arbitrary Lagrangian Eulerian framework. We derive a monolithic variational formulation which allows a robust solution with Newton's method. Temporal discretization is based on the shifted Crank-Nicholson scheme and spatial discretization is done using the finite element method. We validate the numerical implementation on well-known benchmark problems and perform simulations similar to a recent experiment in the literature on a human eye geometry.
\end{abstract}

Keywords: Fluid-Structure Interaction, Viscoelasticity, Finite Element

\section{Introduction}

In this work we consider the interaction between a viscoelastic fluid, modelled by a Burgers-type equation and a hyperelastic solid. To this end we first state the fluid and structure equations in their usual frameworks. Then we derive a monolithic variational formulation of the viscoelastic FSI problem in the arbitrary Lagrangian Eulerian (ALE) framework and summarize the necessary steps for the discretization in time and space. Furthermore we state the interface conditions and the equation for the fluid displacement. We validate the implementation on well-known benchmark problems for Newtonian and viscoelastic FSI. Finally we apply the derived solution method to ophthalmology. We analyse the interaction of the viscoelastic vitreous with its surrounding elastic structures. Given a prescribed displacement on the boundary of the structure we compare the resulting stress distribution in the vitreous for healthy and pathological eyes.

\section{$2 \quad$ Modelling}

First we state the fluid and solid equations in their usual framework. The fluid equations are commonly formulated in Eulerian coordinates. The Burgers-type model has the following form $([1])$

$$
\begin{aligned}
\rho_{f} \partial_{t} v_{f}+\rho_{f}\left(v_{f} \cdot \nabla\right) v_{f}-\operatorname{div} \mathbb{T}=0 & \text { in } \Omega_{f}, t \in I \\
\operatorname{div} v_{f}=0 & \text { in } \Omega_{f}, t \in I \\
\stackrel{\nabla}{B_{1}}+\frac{\mu_{1}}{\nu_{1}}\left(B_{1}-I\right)=0 & \text { in } \Omega_{f}, t \in I \\
\stackrel{\nabla}{B_{2}}+\frac{\mu_{2}}{\nu_{2}}\left(B_{2}-I\right)=0 & \text { in } \Omega_{f}, t \in I
\end{aligned}
$$

with the Cauchy stress tensor

$$
\mathbb{T}=-p_{f} I+\rho_{f} \nu_{3}\left(\nabla v_{f}+\nabla v_{f}^{T}\right)+\mu_{1}\left(B_{1}-I\right)+\mu_{2}\left(B_{2}-I\right)
$$

and the upper convected Oldroyd derivative

$$
\stackrel{\nabla}{S}:=\partial_{t} S+(v \cdot \nabla) S-(\nabla v) S-S(\nabla v)^{T} .
$$

Here $\Omega_{f} \in \mathbb{R}^{n}, n=2,3$ denotes the fluid domain, $v_{f}$ the velocity of the fluid, $\rho_{f}$ its density, $p_{f}$ the pressure and $I=(0, T], T>0$ is the time interval. In addition to viscoelastic fluids we study the Newtonian Navier-Stokes equations which are obtained by dropping the terms involving the tensor-valued unknowns $B_{1}$ and $B_{2}$. 
Next we state the equations for the elastic structure. Let $\hat{\Omega}_{s}$ be the structure domain, $\hat{u}_{s}$ the displacement, $\hat{\rho}_{s}$ the density and $\hat{f}$ an external force. Conservation of momentum in the Lagrangian framework reads

with the first Piola-Kirchhoff stress tensor $\hat{\Pi}$.

$$
\hat{\rho}_{s} \partial_{t}^{2} \hat{u}_{s}-\widehat{\operatorname{div}}(\hat{\Pi})=\hat{\rho}_{s} \hat{f} \quad \text { in } \hat{\Omega}_{s}, t \in I
$$

A simple example for a compressible nonlinear elastic solid is the Saint-Venant-Kirchhoff (STVK) material which is defined by

$$
\hat{\Pi}:=\hat{F}(2 \mu \hat{E}+\lambda \operatorname{tr}(\hat{E}) \hat{I})
$$

with $\hat{E}:=\frac{1}{2}\left(\hat{F}^{T} \hat{F}-\hat{I}\right)$ and $\hat{F}:=\hat{I}+\hat{\nabla} \hat{u}_{s}$. Here $\lambda$ and $\mu$ are the first and second Lamé constants.

For the application to the human eye in Section 4.3 we use the following strain-energy function, which was introduced in [2] and used for the sclera and lens in [3]:

$$
\hat{W}=\frac{1}{2} \mu\left(\hat{J}^{-2 / 3} \operatorname{tr} \hat{C}-3\right)+\frac{1}{2} \kappa(\ln \hat{J})^{2}
$$

with the right Cauchy-Green tensor $\hat{C}=\hat{F}^{T} \hat{F}$ and $\hat{J}=\operatorname{det} \hat{F}$. This yields for the Piola-Kirchhoff tensor

$$
\hat{\Pi}=\frac{\partial \hat{W}}{\partial \hat{F}}=\mu \hat{J}^{-2 / 3}\left(\hat{F}-\frac{1}{3} \operatorname{tr}(\hat{C}) \hat{F}^{-T}\right)+\kappa \ln \hat{J} \hat{F}^{-T} .
$$

The fluid and structure equations have to be supplemented with appropriate initial and boundary conditions.

Using a mixed formulation for the structure equations with $\hat{v}_{s}=\partial_{t} \hat{u}_{s}$ and combining the fluid and structure equations we obtain the following viscoelastic FSI problem:

$$
\begin{aligned}
\rho_{f} \partial_{t} v_{f}+\rho_{f}\left(v_{f} \cdot \nabla\right) v_{f}-\operatorname{div} \mathbb{T} & =\rho_{f} f & & \text { in } \Omega_{f}, t \in I \\
\operatorname{div} v_{f} & =0 & & \text { in } \Omega_{f}, t \in I \\
\partial_{t} B_{1}+\left(v_{f} \cdot \nabla\right) B_{1}-\left(\nabla v_{f}\right) B_{1}-B_{1}\left(\nabla v_{f}\right)^{T}+\frac{\mu_{1}}{\nu_{1}}\left(B_{1}-I\right) & =0 & & \text { in } \Omega_{f}, t \in I \\
\partial_{t} B_{2}+\left(v_{f} \cdot \nabla\right) B_{2}-\left(\nabla v_{f}\right) B_{2}-B_{2}\left(\nabla v_{f}\right)^{T}+\frac{\mu_{2}}{\nu_{2}}\left(B_{2}-I\right) & =0 & & \text { in } \Omega_{f}, t \in I \\
\hat{\rho}_{s} \partial_{t} \hat{v}_{s}-\widehat{\operatorname{div}}(\hat{\Pi}) & =\hat{\rho}_{s} \hat{f}_{s} & & \text { in } \hat{\Omega}_{s}, t \in I \\
\hat{\rho}_{s}\left(\partial_{t} \hat{u}_{s}-\hat{v}_{s}\right) & =0 & & \text { in } \hat{\Omega}_{s}, t \in I
\end{aligned}
$$

with the stress tensors defined as in equation (1) and (2) or (3) depending on the chosen material. This strong form has to be supplemented with appropriate initial, boundary and interface conditions. Here the fluid equations are formulated on moving domains in Eulerian coordinates, while the structure equations are formulated in the Lagrangian framework. In the next chapter we derive the variational formulation on fixed domains employing the ALE transformation.

\section{Discretization}

In the following we state the variational formulation for the viscoelastic FSI problem using the ALE framework [4]. The PDE for the movement of the fluid mesh is a standard harmonic extension in the domain $\hat{\Omega}_{f}$. The interface conditions are continuity of the velocities, displacements and normal stresses. We define the following function spaces

$$
\begin{aligned}
\hat{W}_{f} & :=H^{1}\left(\hat{\Omega}_{f}\right)^{n \times n}, \\
\hat{V}_{f, \hat{v}}^{n, 0} & :=\left\{\hat{v}_{f} \in H_{0}^{1}\left(\hat{\Omega}_{f}\right)^{n}: \hat{v}_{f}=\hat{v}_{s} \text { on } \hat{\Gamma}_{i}\right\}, \\
\hat{V}_{f, \hat{u}}^{n, 0} & :=\left\{\hat{u}_{f} \in H_{0}^{1}\left(\hat{\Omega}_{f}\right)^{n}: \hat{u}_{f}=\hat{u}_{s} \text { on } \hat{\Gamma}_{i}\right\}, \\
\hat{V}_{f, \hat{\Gamma}_{i}}^{n, 0} & :=\left\{\hat{\psi}_{f} \in H_{0}^{1}\left(\hat{\Omega}_{f}\right)^{n}: \hat{\psi}_{f}=\hat{\psi}_{s} \text { on } \hat{\Gamma}_{i}\right\}, \\
\hat{V}_{f, \hat{u}, \hat{\Gamma}_{i}}^{n, 0} & :=\left\{\hat{\psi}_{f} \in H_{0}^{1}\left(\hat{\Omega}_{f}\right)^{n}: \hat{\psi}_{f}=0 \text { on } \hat{\Gamma}_{i}\right\} .
\end{aligned}
$$

Then the variational formulation reads: 
Problem 1 Find $\left\{\hat{v}_{f}, \hat{v}_{s}, \hat{u}_{f}, \hat{u}_{s}, \hat{p}_{f}, \hat{B}_{1}, \hat{B}_{2}\right\} \in\left\{\hat{v}_{f}^{D}+\hat{V}_{f, \hat{v}}^{n, 0}\right\} \times H^{1}\left(\hat{\Omega}_{s}\right)^{n} \times\left\{\hat{u}_{f}^{D}+\hat{V}_{f, \hat{u}}^{n, 0}\right\} \times\left\{\hat{u}_{s}^{D}+\right.$ $\left.H_{0}^{1}\left(\hat{\Omega}_{s}\right)^{n}\right\} \times L^{2}\left(\hat{\Omega}_{f}\right) \backslash \mathbb{R} \times \hat{W}_{f} \times \hat{W}_{f}$ such that $\hat{v}_{f}(0)=\hat{v}_{f}^{0}, \hat{v}_{s}(0)=\hat{v}_{s}^{0}, \hat{u}_{f}(0)=\hat{u}_{f}^{0}, \hat{u}_{s}(0)=\hat{u}_{s}^{0}$, $\hat{B}_{1}(0)=\hat{I}, \hat{B}_{2}(0)=\hat{I}$ and for almost all time steps $t \in I$ it holds:

$$
\begin{aligned}
\left(\hat{J} \hat{\rho}_{f} \partial_{t} \hat{v}_{f}, \hat{\psi}^{v}\right)_{\hat{\Omega}_{f}}+\left(\hat{\rho}_{f} \hat{J}\left(\hat{F}^{-1}\left(\hat{v}_{f}-\partial_{t} \hat{u}_{f}\right) \cdot \hat{\nabla}\right) \hat{v}_{f}, \hat{\psi}^{v}\right)_{\hat{\Omega}_{f}} & \\
+\left(\hat{J} \hat{\mathbb{T}} \hat{F}^{-T}, \hat{\nabla} \hat{\psi}^{v}\right)_{\hat{\Omega}_{f}}-\left(\hat{\rho}_{f} \hat{J} \hat{f}_{f}, \hat{\psi}^{v}\right)_{\hat{\Omega}_{f}} & =0 \forall \hat{\psi}^{v} \in \hat{V}_{f, \hat{\Gamma}_{i}}^{n, 0}, \\
\left(\hat { J } \left(\partial_{t} \hat{B}_{1}+\left(\hat{\nabla} \hat{B}_{1}\right)\left(\hat{F}^{-1}\left(\hat{v}_{f}-\partial_{t} \hat{u}_{f}\right)\right)-\left(\hat{\nabla} \hat{v}_{f}\right) \hat{F}^{-1} \hat{B}_{1}-\hat{B}_{1} \hat{F}^{-T}\left(\hat{\nabla} \hat{v}_{f}\right)^{T}\right.\right. & \\
\left.\left.+\frac{\mu_{1}}{\nu_{1}}\left(\hat{B}_{1}-\hat{I}\right)\right), \hat{\psi}^{B_{1}}\right)_{\hat{\Omega}_{f}} & =0 \forall \hat{\psi}^{B_{1}} \in \hat{W}_{f}, \\
\left(\hat { J } \left(\partial_{t} \hat{B}_{2}+\left(\hat{\nabla} \hat{B}_{2}\right)\left(\hat{F}^{-1}\left(\hat{v}_{f}-\partial_{t} \hat{u}_{f}\right)\right)-\left(\hat{\nabla} \hat{v}_{f}\right) \hat{F}^{-1} \hat{B}_{2}-\hat{B}_{2} \hat{F}^{-T}\left(\hat{\nabla} \hat{v}_{f}\right)^{T}\right.\right. & \\
\left.\left.+\frac{\mu_{2}}{\nu_{2}}\left(\hat{B}_{2}-\hat{I}\right)\right), \hat{\psi}^{B_{2}}\right)_{\hat{\Omega}_{f}} & =0 \forall \hat{\psi}^{B_{2}} \in \hat{W}_{f}, \\
\left(\hat{\rho}_{s} \partial_{t} \hat{v}_{s}, \hat{\psi}^{v}\right)_{\hat{\Omega}_{s}}+\left(\hat{\Pi}, \hat{\nabla} \hat{\psi}^{v}\right)_{\hat{\Omega}_{s}}-\left\langle\hat{\Pi} \hat{n}_{s}, \hat{\psi}^{v}\right\rangle_{\hat{\Gamma}_{N}}-\left(\hat{\rho} \hat{f}_{s}, \hat{\psi}^{v}\right)_{\hat{\Omega}_{s}} & =0 \forall \hat{\psi}^{v} \in H_{0}^{1}\left(\hat{\Omega}_{s}\right)^{n}, \\
\hat{\rho}_{s}\left(\partial_{t} \hat{u}_{s}-\hat{v}_{s}, \hat{\psi}^{u}\right)_{\hat{\Omega}_{s}} & =0 \forall \hat{\psi}^{u} \in H^{1}\left(\hat{\Omega}_{s}\right)^{n}, \\
\left(\widehat{\operatorname{div}}\left(\hat{J} \hat{F}^{-1} \hat{v}_{f}\right), \hat{\psi}^{p}\right)_{\hat{\Omega}_{f}} & =0 \forall \hat{\psi}^{p} \in L^{2}\left(\hat{\Omega}_{f}\right) \backslash \mathbb{R}, \\
\left(\hat{\alpha}_{u} \hat{\nabla}_{u_{f}}, \hat{\nabla} \hat{\psi}^{u}\right)_{\hat{\Omega}_{f}} & =0 \forall \hat{\psi}^{u} \in \hat{V}_{f, \hat{u}, \hat{\Gamma}_{i}}^{n, 0}
\end{aligned}
$$

with

$$
\hat{\mathbb{T}}=-\hat{p}_{f} \hat{I}+\hat{\rho}_{f} \nu_{3}\left(\hat{\nabla} \hat{v}_{f} \hat{F}^{-1}+\hat{F}^{-T} \hat{\nabla} \hat{v}_{f}^{T}\right)+\mu_{1}\left(\hat{B}_{1}-\hat{I}\right)+\mu_{2}\left(\hat{B}_{2}-\hat{I}\right) .
$$

Here $(\cdot, \cdot)_{\hat{\Omega}_{f}}$ is the usual $L^{2}\left(\hat{\Omega}_{f}\right)$ product. For details on the derivation of the variational formulation using the Navier-Stokes equations we refer to [5] and [6]. The extension to viscoelastic fluids works analogously.

Temporal discretization is done using the shifted Crank-Nicholson scheme [7]. For the finite elements we choose $Q_{2}$ elements for the velocity, displacement and viscoelastic tensors and $P_{1}^{\text {disc }}$ elements for the pressure. The combination of velocity and pressure space satisfies the inf-sup condition. For the linearization we use Newton's method with exact Jacobians.

\section{Numerical Results}

The numerical simulations are realized in deal.ii [8] and are based on the FSI implementation in [9].

\subsection{Stationary viscoelastic FSI}

We start by validating the viscoelastic FSI implementation on well-known benchmarks. First we study the stationary FSI1 benchmark from [10] using the Burgers model instead of the NavierStokes equations for the fluid flow. The benchmark consists of a channel with an inflow from the left and a circular obstacle. In addition there is an elastic beam attached to the obstacle (Figure 1). The parabolic inflow velocity on $\hat{\Gamma}_{\text {in }}$ is

$$
v(0, y)=\left(1.5 \bar{v} \frac{y(0.41-y)}{\left(\frac{0.41}{2}\right)^{2}}, 0\right)
$$

with $\bar{v}=0.2$. For the viscoelastic tensors the boundary condition has to be chosen accordingly on $\hat{\Gamma}_{i}$ (see [11]). Furthermore we have the no slip condition for the velocity on $\hat{\Gamma}_{\text {wall }}$ and the donothing condition on $\hat{\Gamma}_{\text {out }}$. For the structure we use the STVK material (2). Material parameters 
are chosen as

$$
\rho_{s}=\rho_{f}=1000, \quad \nu_{3}=10^{-3}, \quad \mu_{s}=0.5 \cdot 10^{6}, \quad \lambda_{s}=2 \cdot 10^{6}, \quad \mu_{1}=\mu_{2}=25, \quad \nu_{1}=\nu_{2}=0.125 .
$$

This setup is equivalent to the more common Oldroyd-B model with $\mu=50$ and $\nu=0.25$.

The drag and lift coefficients are given by

$$
\begin{aligned}
J_{\text {drag }} & :=\int_{\hat{S}}\left(\hat{J} \hat{\mathbb{T}} \hat{F}^{-T}\right) \hat{n}_{f} \cdot e_{1} \mathrm{~d} \hat{s} \\
J_{\text {lift }} & :=\int_{\hat{S}}\left(\hat{J} \hat{\mathbb{T}} \hat{F}^{-T}\right) \hat{n}_{f} \cdot e_{2} \mathrm{~d} \hat{s}
\end{aligned}
$$

with $\hat{S}:=\hat{\Gamma}_{\text {flag }} \cup\left(\hat{\Gamma}_{\text {circle }} \backslash \hat{\Gamma}_{\text {base }}\right)$.

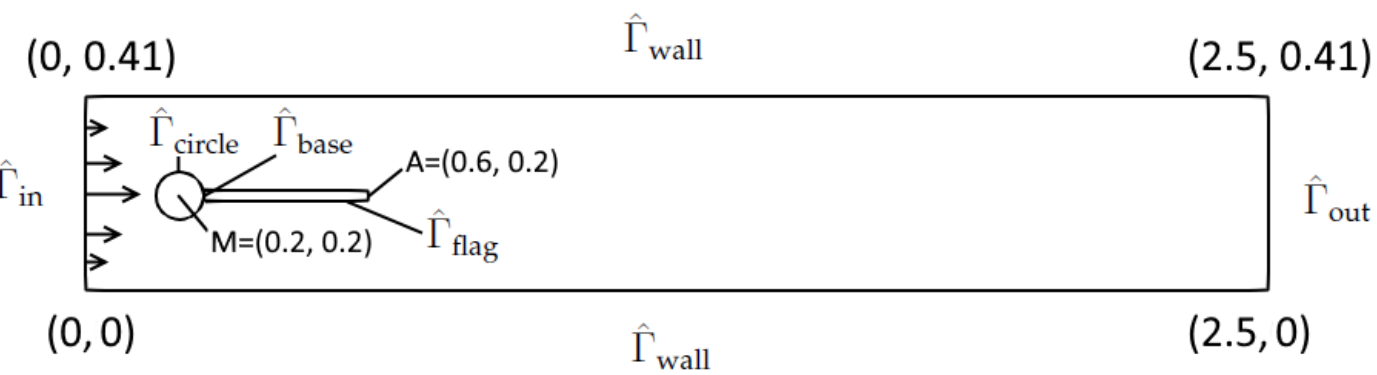

Figure 1: Setup for the FSI benchmarks.

Figure 2 shows the convergence of the drag and lift. The convergence order for global refinement is not optimal because of the limited regularity due to the reentrant corner of the beam [6]. In addition to global refinement we use the dual-weighted residual method from [12] similar to the FSI approach in [5] for Newtonian fluids. This adaptive refinement strategy yields a faster convergence in the drag and lift functionals. Due to the lack of knowledge of the exact reference values the convergence in the last step of the adaptive refinement might be overestimated.
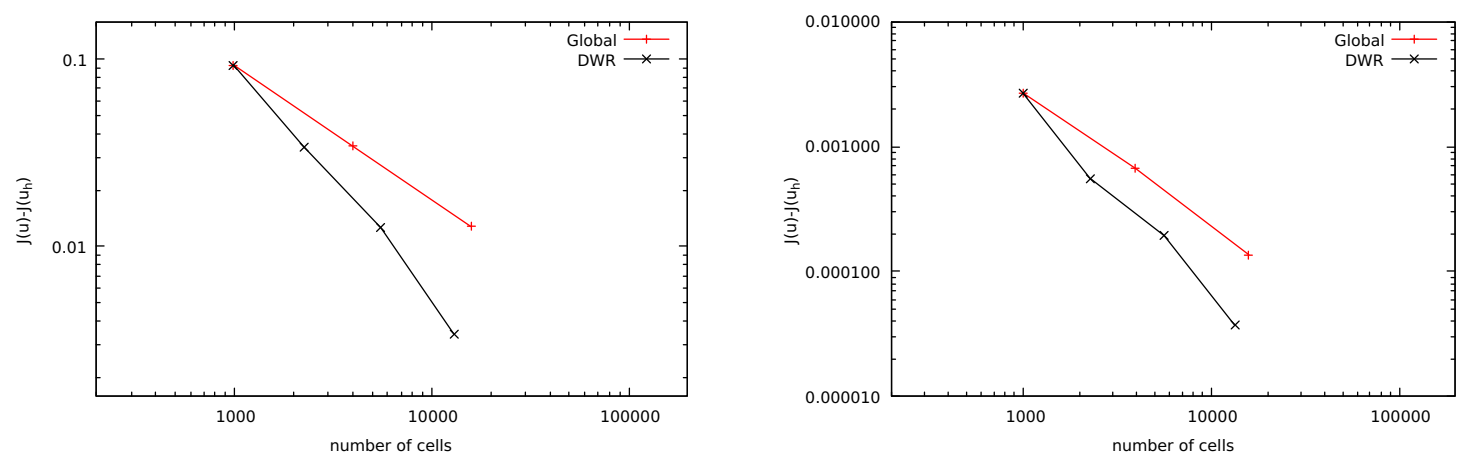

Figure 2: Convergence of the drag (left) and the lift (right) for the viscoelastic FSI1 benchmark.

\subsection{Time dependent viscoelastic FSI}

Before studying time dependent viscoelastic FSI we validate the Newtonian FSI implementation on the non-stationary FSI3 benchmark introduced in [10]. The setup is similar to the FSI1 benchmark. The parabolic inflow on $\hat{\Gamma}_{\text {in }}$ is

$$
v(0, y)=\left(1.5 \bar{v} \frac{y(0.41-y)}{\left(\frac{0.41}{2}\right)^{2}}, 0\right)
$$


with a smooth increase in the beginning

$$
v(t, 0, y)= \begin{cases}v(0, y) \frac{1-\cos \left(\frac{\pi}{2} t\right)}{2} & \text { if } t<2.0 \\ v(0, y) & \text { else. }\end{cases}
$$

The parameters are chosen as

$$
\rho_{f}=\rho_{s}=10^{3}, \quad \nu_{3}=10^{-3}, \quad \mu_{s}=2 \cdot 10^{6}, \quad \lambda_{s}=8 \cdot 10^{6}
$$

and $\bar{v}=2.0$. This yields a Reynolds number of $\mathrm{Re}=200$. The quantities of interest are the displacement at the point $A=(0.6,0.2)$ (see Figure 1 ), the drag and the lift.

Table 1 shows the computed values for three different mesh sizes and two different timesteps. The results are in very good agreement with the available literature [10], [13].

Table 1: Results for the quantities of interest on three different mesh levels with 18928, 75712 and 302848 degrees of freedom with different timestep size $\Delta t$ for FSI3 with Navier-Stokes.

\begin{tabular}{|l|l|l|l|l|l|}
\hline dofs & $\Delta t$ & $\mathrm{x}$-disp $\cdot 10^{-3}$ & $\mathrm{y}$-disp $\cdot 10^{-3}$ & drag & lift \\
\hline 18928 & 0.001 & $-2.82 \pm 2.67$ & $1.50 \pm 34.24$ & $451.56 \pm 22.75$ & $2.11 \pm 157.39$ \\
\hline 75712 & 0.001 & $-2.80 \pm 2.64$ & $1.47 \pm 34.37$ & $457.49 \pm 25.35$ & $2.23 \pm 155.89$ \\
\hline 302848 & 0.001 & $-2.85 \pm 2.69$ & $1.47 \pm 34.76$ & $459.57 \pm 26.86$ & $2.14 \pm 158.90$ \\
\hline 18928 & 0.0005 & $-2.83 \pm 2.67$ & $1.50 \pm 34.24$ & $451.45 \pm 22.66$ & $2.14 \pm 156.75$ \\
\hline 75712 & 0.0005 & $-2.80 \pm 2.64$ & $1.47 \pm 34.36$ & $457.38 \pm 25.28$ & $2.32 \pm 155.07$ \\
\hline 302848 & 0.0005 & $-2.85 \pm 2.69$ & $1.46 \pm 34.75$ & $459.47 \pm 26.80$ & $2.23 \pm 157.98$ \\
\hline ref. $[13]$ & & $-2.88 \pm 2.72$ & $1.47 \pm 34.99$ & $460.5 \pm 27.74$ & $2.50 \pm 153.9$ \\
\hline
\end{tabular}

Next we study the FSI3 benchmark using the Burgers model. The parameters are chosen as in the previous example with the additional parameters for the Burgers model chosen as $\mu_{1}=\mu_{2}=50$ and $\nu_{1}=\nu_{2}=0.25$. Figure 3 shows the velocity magnitude in the current fluid domain $\Omega_{f}$ at three different times.
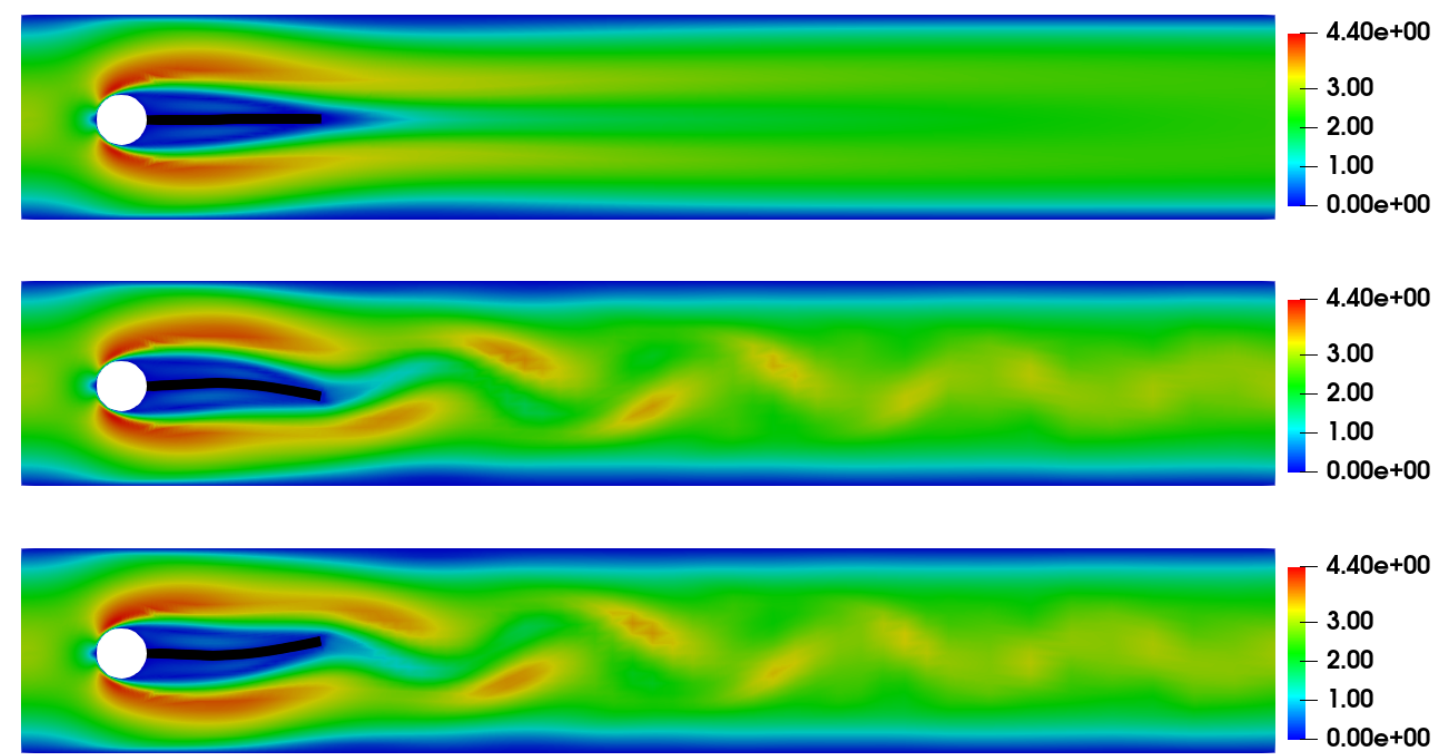

Figure 3: Velocity magnitude in $\Omega_{f}$ at three different times for the viscoelastic FSI3 case

Figure 4 shows the plots for the quantities of interest in the time interval $[9,10]$. The mean for the drag is higher and the amplitude lower than in the Navier-Stokes case. For the lift the 
mean and amplitude are smaller than in the Navier-Stokes case. The y-displacement shows a similar behaviour to the lift in comparison to the Navier-Stokes variant. Finally Table 2 shows the convergence of the mean and amplitude of the quantities of interest for different refinement levels and timestep sizes. The frequencies for the viscoelastic and Newtonian case are very similar: For the viscoelastic case the frequencies are $f_{1}=10.84$ and $f_{2}=5.42$ on the finest mesh. For the Navier-Stokes case the frequencies on the finest mesh are $f_{1}=10.99 f_{2}=5.49$.
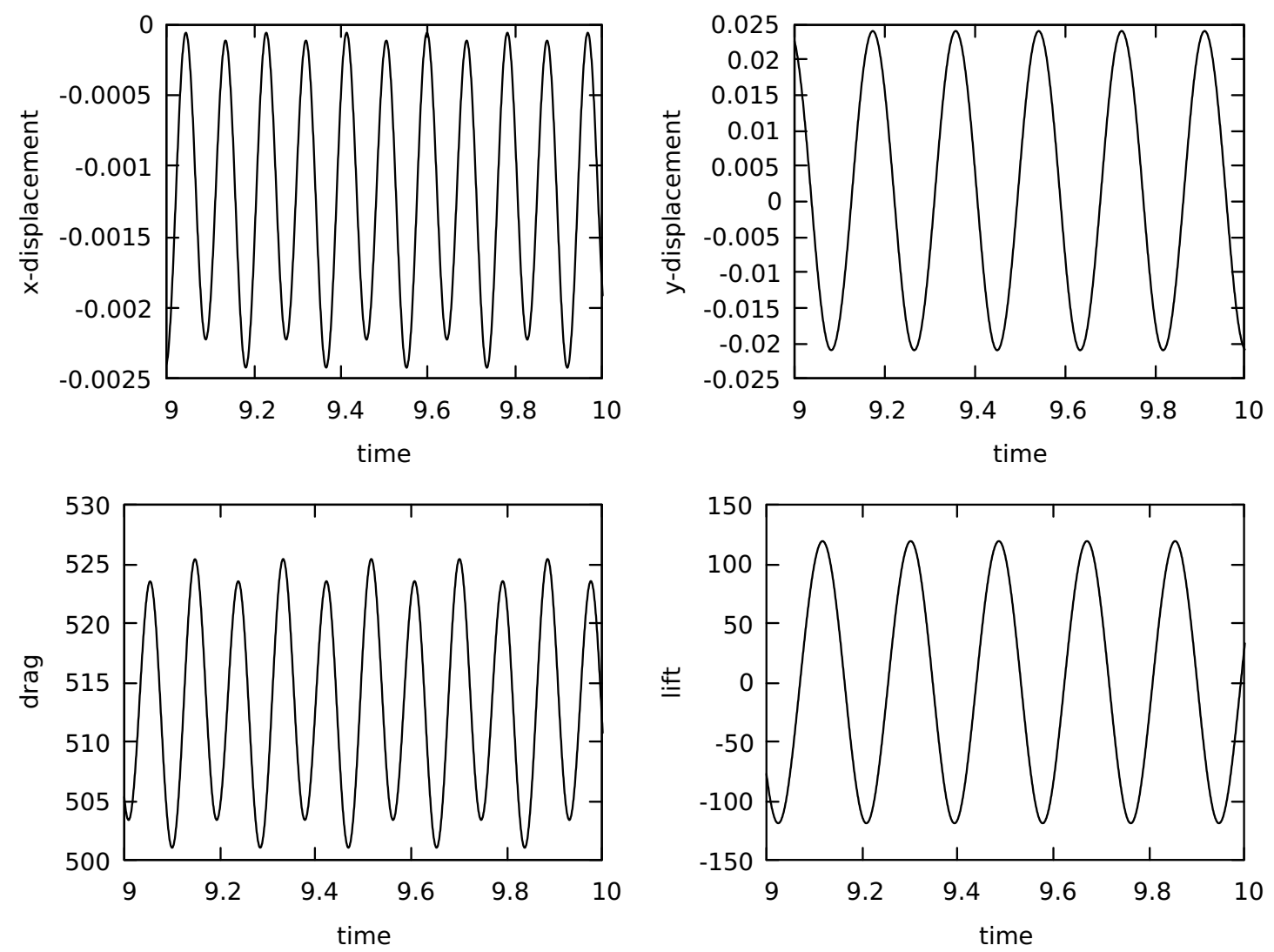

Figure 4: Plots of the four quantities of interest over time for the viscoelastic FSI3 case

Table 2: Results for the quantities of interest on three different mesh levels with 7543, 30172 and 120688 degrees of freedom with different timestep size $\Delta t$ for the viscoelastic FSI3 case.

\begin{tabular}{|l|l|l|l|l|l|}
\hline dofs & $\Delta t$ & $\mathrm{x}$-disp $\cdot 10^{-3}$ & $\mathrm{y}$-disp $\cdot 10^{-3}$ & drag & lift \\
\hline 10354 & 0.001 & $-1.78 \pm 1.73$ & $1.21 \pm 27.52$ & $513.14 \pm 15.45$ & $6.76 \pm 154.57$ \\
\hline 41416 & 0.001 & $-1.13 \pm 1.07$ & $1.57 \pm 21.31$ & $509.86 \pm 10.33$ & $0.42 \pm 115.75$ \\
\hline 165664 & 0.001 & $-1.25 \pm 1.18$ & $1.53 \pm 22.53$ & $513.63 \pm 12.29$ & $0.69 \pm 119.61$ \\
\hline 10354 & 0.0005 & $-1.79 \pm 1.73$ & $1.20 \pm 27.53$ & $513.29 \pm 15.38$ & $6.80 \pm 154.05$ \\
\hline 41416 & 0.0005 & $-1.13 \pm 1.07$ & $1.57 \pm 21.28$ & $509.65 \pm 10.25$ & $0.44 \pm 115.46$ \\
\hline 165664 & 0.0005 & $-1.24 \pm 1.18$ & $1.53 \pm 22.48$ & $513.29 \pm 12.19$ & $0.75 \pm 119.14$ \\
\hline
\end{tabular}

\subsection{Applications in ophthalmology}

Finally we consider applications in ophthalmology. The setup is similar to a recent experiment on bovine eyes [14]. We use a simplified human eye geometry cut in the middle of the eye similar to the experiment (see Figure 5). The blue part is the fluid domain while the grey and red part are the elastic structures. The eye is fixed on the left and pulled to the right leading to a deformation of 
the eye. Similar simulations were performed on a bovine eye mesh using low-order finite elements to reduce the numerical costs in [15]. The displacement magnitude can be seen in Figure 5. Figure 6 shows the norm of the average stress per cell for a healthy viscoelastic vitreous on the left and on the right for a pathological vitreous using the Navier-Stokes equations as fluid model. The stress in the healthy eye is higher by almost a factor of eight. This is an interesting result since some medical diseases in the eye are possibly linked to the stress distribution in the vitreous.
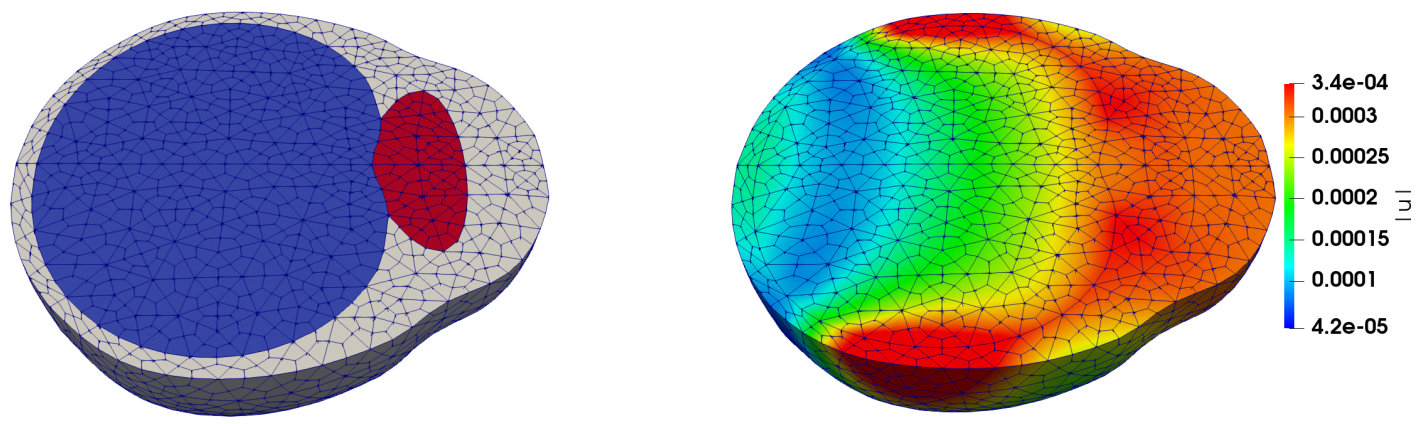

Figure 5: Left: mesh with 11568 cells with 1175422 degrees of freedom (304053 (velocity) +304053 (displacement) +20704 (pressure) +546612 (viscoelastic tensors)). Right: displacement magnitude.
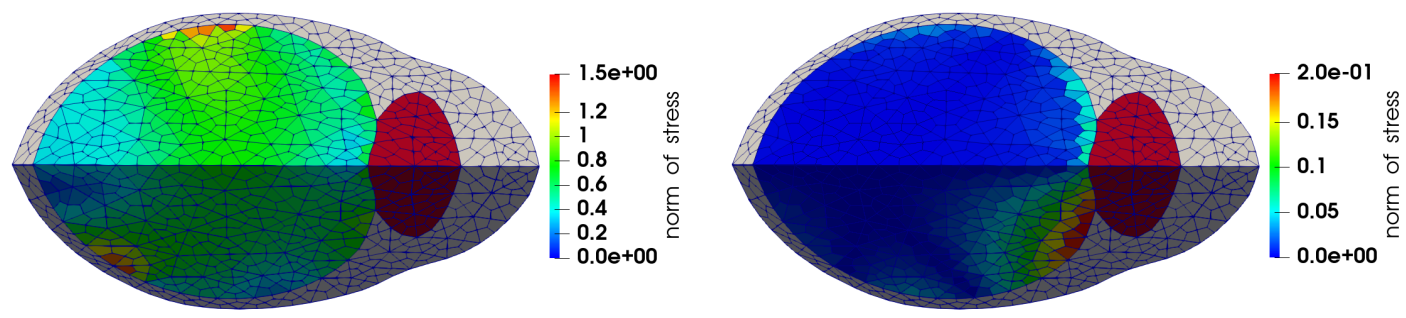

Figure 6: Stress tensor in the vitreous for Burgers (left) and Navier-Stokes (right) sliced in the middle for visualization purposes.

\section{Conclusion}

In this work we validated our viscoelastic FSI implementation on two typical benchmark problems by studying the convergence for different functionals of interest for different mesh and timestep sizes. The simulations showed that the numerical approach for viscoelastic fluids in the ALE framework works well for problems with large deformations and high Reynolds number. The simulations showed that for this set of parameters for $\mu_{1}, \mu_{2}, \nu_{1}$ and $\nu_{2}$ the viscoelasticity has a big impact on the quantities of interest in comparison to the Navier-Stokes case. Nevertheless investigation of different sets of parameters might be interesting. Due to the fast movement of the elastic beam it might also be necessary to study stabilization methods.

Furthermore we performed simulations on a human eye geometry to study the behaviour of the vitreous for healthy and pathological eyes. We observed big differences in the stress distribution in the vitreous with a higher stress magnitude in the healthy case.

\section{Acknowledgment}

The financial support for this project is provided by the Klaus Tschira Stiftung gGmbH, Project No.00.265.2015. 


\section{References}

[1] J. Hron, K. Rajagopal, and K. Tůma: Flow of a Burgers fluid due to time varying loads on deforming boundaries, Journal of Non-Newtonian Fluid Mechanics 210, 66-77 (2014).

[2] J. Simo, R. L. Taylor, and K. Pister: Variational and projection methods for the volume constraint in finite deformation elasto-plasticity, Computer methods in applied mechanics and engineering 51(1-3), 177-208 (1985).

[3] R. Grytz, M. A. Fazio, M. J. Girard, V. Libertiaux, L. Bruno, S. Gardiner, C. A. Girkin, and J. C. Downs: Material properties of the posterior human sclera, Journal of the mechanical behavior of biomedical materials 29, 602-617 (2014).

[4] J. Donea, S. Giuliani, and J.P. Halleux: An arbitrary Lagrangian-Eulerian finite element method for transient dynamic fluid-structure interactions, Computer methods in applied mechanics and engineering 33(1-3), 689-723 (1982).

[5] T. Wick: Adaptive finite element simulation of fluid-structure interaction with application to heart-valve dynamics, $\mathrm{PhD}$ thesis, University of Heidelberg, 2011.

[6] T. Richter: Fluid-structure Interactions: Models, Analysis and Finite Elements (Springer, 2017).

[7] J. G. Heywood and R. Rannacher: Finite-element approximation of the nonstationary navierstokes problem. part iv: Error analysis for second-order time discretization, SIAM Journal on Numerical Analysis 27(2), 353-384 (1990).

[8] W. Bangerth, R. Hartmann, and G. Kanschat: deal.II - a general purpose object oriented finite element library, ACM Trans. Math. Softw. 33(4), 24/1-24/27 (2007).

[9] T. Wick: Solving monolithic fluid-structure interaction problems in arbitrary Lagrangian Eulerian coordinates with the deal. ii library, Archive of Numerical Software 1(1), 1-19 (2013).

[10] S. Turek and J. Hron: Proposal for numerical benchmarking of fluid-structure interaction between an elastic object and laminar incompressible flow, in: Fluid-structure interaction, (Springer, 2006), pp. 371-385.

[11] H. S. Dou and N. Phan-Thien: The flow of an Oldroyd-B fluid past a cylinder in a channel: adaptive viscosity vorticity (davss- $\omega$ ) formulation, Journal of Non-Newtonian Fluid Mechanics 87(1), 47-73 (1999).

[12] W. Bangerth and R. Rannacher: Adaptive finite element methods for differential equations (Birkhäuser, 2013).

[13] S. Turek, J. Hron, M. Razzaq, H. Wobker, and M. Schäfer: Numerical benchmarking of fluid-structure interaction: A comparison of different discretization and solution approaches, in: Fluid Structure Interaction II, (Springer, 2011), pp. 413-424.

[14] N.S. Shah, D.C. Beebe, S.P. Lake, and B. A. Filas: On the spatiotemporal material anisotropy of the vitreous body in tension and compression, Annals of biomedical engineering 44(10), 3084-3095 (2016).

[15] K. Tůma, J. Stein, V. Průša, and E. Friedmann: Motion of the vitreous humour in a deforming eye-fluid-structure interaction between a nonlinear elastic solid and a nonlinear viscoleastic fluid, arXiv preprint arXiv:1802.04002 (2018). 